\title{
No evidence for PALB2 methylation in high-grade serous ovarian cancer
}

\author{
Thomas Mikeska ${ }^{1,2+}$, Kathryn Alsop ${ }^{3,4+}$, Australian Ovarian Cancer Study Group ${ }^{3}$, Gillian Mitchell ${ }^{5}$, \\ David DL Bowtell ${ }^{2,3,4,6}$ and Alexander Dobrovic ${ }^{1,2,6^{*}}$
}

\begin{abstract}
Background: High-grade serous ovarian cancers are a distinct histological subtype of ovarian cancer often characterised by a dysfunctional BRCA/Fanconi anaemia (BRCA/FA) pathway, which is critical to the homologous recombination DNA repair machinery. An impaired BRCA/FA pathway sensitises tumours to the treatment with DNA cross-linking agents and to PARP inhibitors. The vast majority of inactivating mutations in the BRCA/FA pathway are in the BRCA1 and BRCA2 genes and occur predominantly in high-grade serous cancer. Another member of the BRCA/FA pathway, PALB2 (FANCN), was reported to have been inactivated by DNA methylation in some sporadic ovarian cancers. We therefore sought to investigate the role of PALB2 methylation in high-grade serous ovarian cancers.
\end{abstract}

Finding: PALB2 methylation was investigated in 92 high-grade serous ovarian cancer samples using methylationsensitive high-resolution melting analysis. DNA methylation of PALB2 was not detected in any of the ovarian cancer samples investigated.

Conclusion: Epigenetic silencing by DNA methylation of PALB2 is not a common event in high-grade serous ovarian cancers.

Keywords: DNA methylation, Ovarian cancer, Fanconi anaemia, PALB2, MS-HRM

\section{Findings}

Ovarian cancer comprises several broad groups of distinct diseases [1]. The largest group are high-grade serous ovarian cancers, of which a substantial proportion are characterised by an impaired BRCA/Fanconi anaemia (BRCA/FA) pathway [2,3]. The BRCA/FA pathway is a key part of the homologous recombination DNA repair machinery and includes the BRCA1 and BRCA2 genes as well as members of the Fanconi anaemia complementation group. The inactivation of the BRCA/FA pathway is associated with an increased sensitivity of cancerous cells to DNA cross-linking agents and to PARP inhibitors $[4,5]$.

The vast majority of inactivating mutations that occur in the BRCA/FA pathway in high-grade serous ovarian

\footnotetext{
* Correspondence: Alexander.Dobrovic@petermac.org

${ }^{\dagger}$ Equal contributors

'Molecular Pathology Research and Development Laboratory, Department of Pathology, Peter MacCallum Cancer Centre, East Melbourne, VIC, Australia ${ }^{2}$ Department of Pathology, The University of Melbourne, Parkville, VIC, Australia

Full list of author information is available at the end of the article
}

carcinomas are found in the BRCA1 and BRCA2 genes [2]. The protein product of the Fanconi anaemia gene PALB2 (FANCN) serves as a bridge between BRCA1 and BRCA2 [6]. Mutations of PALB2 have been associated with familial breast cancer and pancreatic cancer [6]. The occurrence of PALB2 mutations in ovarian cancer has been less studied but is probably rare $[7,8]$.

Aberrant DNA methylation is an alternative mechanism for PALB2 inactivation. PALB2 methylation has been reported in familial and sporadic breast cancer cases as well as in sporadic ovarian cancer samples [9]. In the sporadic ovarian cancer samples, PALB2 methylation was reported to occur at a frequency of approximately $8 \%$. However, the number of sporadic ovarian cancer cases investigated was quite small (53 samples) and consisted of different histological subtypes, grades and stages.

We sought to investigate aberrant PALB2 methylation in a large number of high-grade serous ovarian cancers using methylation-sensitive high-resolution melting (MS-HRM) [10]. MS-HRM uses methylation-independent PCR primers which allow the amplification of bisulfite-

\section{Biomed Central}


modified templates independent of their methylation status. The analysis is based on the different melting behaviour of unmethylated and methylated templates after PCR amplification. The melting behaviour of an individual sample is visualised as a melting profile, which can be compared to melting profiles of DNA methylation standards and allows the estimation of the amount of methylation semi-quantitatively [11].

Ninety-two unselected high-grade serous ovarian cancer samples from The Australian Ovarian Cancer Study (AOCS) were used in this study. AOCS is a populationbased case control study where newly diagnosed cases of ovarian, peritoneal and fallopian tube tumours were prospectively ascertained from major treatment centres and state-based cancer registries around Australia between January 2002 and June 2006, as previously described $[3,12]$. DNA was extracted from the fresh-frozen primary tumour samples using the DNeasy Blood and Tissue Kit (Qiagen, Hilden, Germany). Primary tissue sample assessed as being of low tumour content by pathological review was needle macro-dissected before DNA extraction. DNA concentration and quality was measured using the NanoDrop ND-1000 Spectrophotometer (NanoDrop Technologies, Thermo Fisher Scientific, Wilmington, DE). The use of the DNA has been approved by the Human Research Ethics Committee at the Peter MacCallum Cancer Centre. Fully methylated human control DNA was obtained commercially (Millipore, Billerica, MA). Control DNAs from the peripheral blood of normal individuals and the HL-60 cell line were extracted by using the QIAamp DNA Blood Mini Kit (Qiagen) according to the manufacturer's instructions.

For bisulfite modification, $200 \mathrm{ng}$ of DNA extracted from the high-grade serous ovarian cancer samples and $500 \mathrm{ng}$ of the control DNAs were bisulfite modified using the EpiTect Bisulfite Kit (Qiagen) according to the manufacturer's instructions. The bisulfite-modified DNA from the high-grade serous ovarian cancer samples was eluted twice in a final volume of $40 \mu \mathrm{L}$ ( $50 \mu \mathrm{L}$ for the control DNAs) of the supplied elution buffer, to give a theoretical concentration of $5 \mathrm{ng} / \mu \mathrm{L}(10 \mathrm{ng} / \mu \mathrm{L}$ for the control DNAs) presuming no loss of DNA during bisulfite modification.
PALB2 and RASSF1A methylation was investigated by MS-HRM. DNA methylation standard series were prepared by diluting the bisulfite-modified fully methylated control DNA in bisulfite-modified unmethylated control DNA from peripheral blood for the analysis of PALB2 methylation, and from HL-60 for the analysis of RASSF1A methylation, respectively. The amount of PCR amplifiable templates of the fully methylated and unmethylated control DNAs was normalised prior to dilution as previously described [13]. The DNA methylation standard series comprised $100 \%$, $50 \%, 25 \%, 10 \%$, and $0 \%$ of methylated control DNA. MSHRM was performed on a Rotor-Gene 6000 (Corbett, Sydney, Australia). Each sample and each DNA methylation standard was run in duplicate, while the genomic DNA control and the no template control were run only once.

The PCR primer sequences for the PALB2 methylation

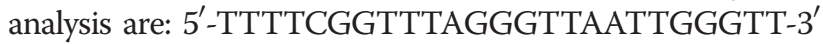
(forward primer) and 5'-CACCTTTTCCTTCTCCTCAC AACTAAA-3' (reverse primer). The PCR amplicon is $135 \mathrm{bp}$ in size and corresponds to GenBank accession number AC008870.8, nucleotides 68,324 to 68,458 (UCSC Genome Browser: chr16: 23,652,428 to 23,652,562; GRCh37/hg19) (Figure 1). The amplicon contains 10 CpG dinucleotides and a G/A single-nucleotide polymorphism (rs8053188) between the primers.

PCR was performed in $0.1 \mathrm{~mL}$ tubes with a final reaction volume of $20 \mu \mathrm{L}$ containing $200 \mathrm{nmol} / \mathrm{L}$ of the forward primer, $400 \mathrm{nmol} / \mathrm{L}$ of the reverse primer, $200 \mu \mathrm{mol} / \mathrm{L}$ of each dNTP, $5 \mu \mathrm{mol} / \mathrm{L}$ SYTO 9 (Life Technologies, Carlsbad, CA), $3.5 \mathrm{mmol} / \mathrm{L} \mathrm{MgCl}_{2}, 0.5 \mathrm{U}$ HotStarTaq DNA polymerase in its supplied buffer (1X) (Qiagen) and $5 \mathrm{ng}$ (10 ng for the DNA methylation standard series) of bisulfite-modified DNA. PCR amplification was performed with one cycle of $95^{\circ} \mathrm{C}$ for $15 \mathrm{~min}$, 50 cycles of $95^{\circ} \mathrm{C}$ for $20 \mathrm{~s}, 62^{\circ} \mathrm{C}$ for $20 \mathrm{~s}$ and $72^{\circ} \mathrm{C}$ for $30 \mathrm{~s}$. This was immediately followed by a hold at $95^{\circ} \mathrm{C}$ for $1 \mathrm{~min}, 70^{\circ} \mathrm{C}$ for $1.5 \mathrm{~min}$ and a HRM step from 70 to $95^{\circ} \mathrm{C}$ rising at $0.2^{\circ} \mathrm{C}$ per second, and holding for $1 \mathrm{~s}$ after each stepwise increment. RASSF1A methylation analysis was performed as previously described [14] with an altered PCR amplification profile: one cycle of $95^{\circ} \mathrm{C}$ for $15 \mathrm{~min}, 55$ cycles of $95^{\circ} \mathrm{C}$ for $10 \mathrm{~s}, 65^{\circ} \mathrm{C}$ for $20 \mathrm{~s}$ and $72^{\circ} \mathrm{C}$ for $30 \mathrm{~s}$. This was immediately followed by a hold

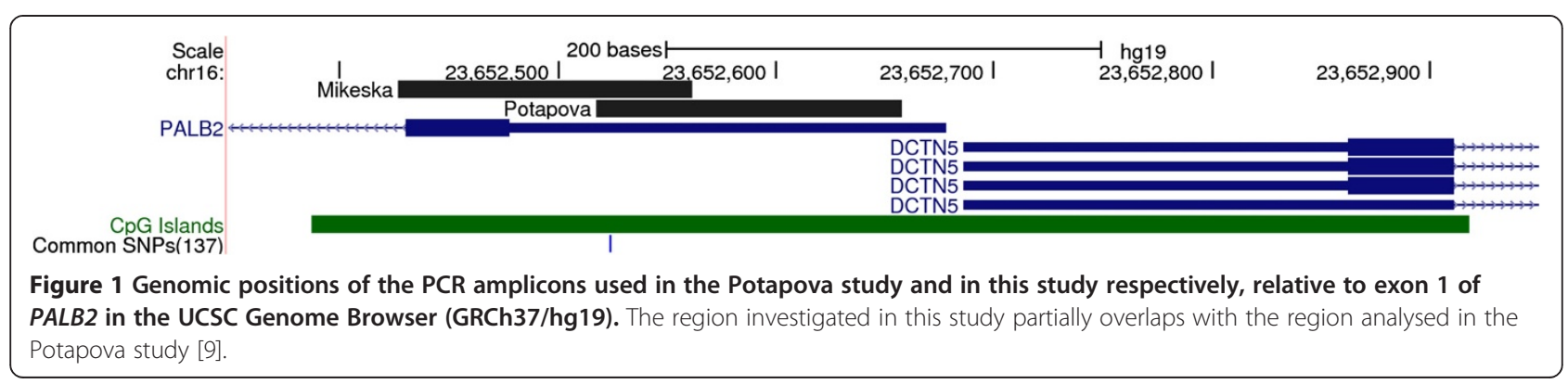


at $97^{\circ} \mathrm{C}$ for $1 \mathrm{~min}, 65^{\circ} \mathrm{C}$ for $1.5 \mathrm{~min}$ and a HRM step from 65 to $95^{\circ} \mathrm{C}$ rising at $0.2^{\circ} \mathrm{C}$ per second, and holding for $1 \mathrm{~s}$ after each stepwise increment.

The 92 high-grade serous ovarian cancer samples were investigated for aberrant PALB2 methylation in exon 1 using MS-HRM. DNA methylation was not detectable in any of the high-grade serous ovarian cancer samples (Figure 2). To ensure that methylation would be detected if present, the samples were also tested for RASSF1A methylation which has been previously reported in highgrade serous ovarian cancer [15]. RASSF1A methylation was seen at various levels in a large proportion of the samples: 24 samples (26\%) had no methylation, 46 samples (50\%) had less than $10 \%$ methylation and 22 samples (24\%) had methylation levels ranging from $10 \%$ to $90 \%$.

Potapova et al. reported methylation of a region in exon 1 of PALB2 in breast and ovarian cancers [9]. Quantitative methylation-specific PCR was used to detect aberrant PALB2 methylation. DNA methylation of the methylation-positive samples was subsequently confirmed by direct bisulfite sequencing. The region investigated by MS-HRM partially overlaps with the above region and contains the two $\mathrm{CpG}$ dinucleotides of the reverse methylation-specific PCR (MSP) primer used in the previous study (Figure 1). A positive MSP result can not be obtained without one or both of those CpG dinucleotides being methylated. Thus our results have not been compromised by analysing an incompletely overlapping region to that previously analysed, which is a necessary consequence of the different PCR primer design principles for the two assays.

MS-HRM is a reliable and sensitive methodology which is well suited for the detection of homogeneous and heterogeneous methylation at gene-specific loci $[16,17]$. The PALB2 MS-HRM assay conditions have been optimised and show sensitivity for the reliable detection of methylated epialleles down to below $10 \%$ (Figure 2). The sensitivity of the MS-HRM assay to detect aberrant DNA methylation is sufficient as it was estimated that some samples showed less than 20\% of methylated epialleles [9].

Interestingly, all the four sporadic ovarian cancer cases that were found to be positive for PALB2 methylation in the Potapova study were clear cell carcinomas, or showed foci of clear cell carcinoma [9]. Clear cell ovarian tumours have been compared to renal cell tumours in the past [18], and are now believed to be not only morphologically [19], but molecularly distinct when compared to high-grade serous and high-grade endometrioid ovarian cancers $[20,21]$.

The lack of PALB2 methylation-positive samples in our study might be explained by the fact that we have investigated high-grade serous ovarian cancer cases only, which presumably evolve via a different tumorigenic pathway of development than clear cell carcinomas [20]. However, because high-grade serous ovarian cancer is driven by disrupted homologous recombination, we may have reasonably expected to observe PALB2 methylation as a method of pathway disruption.

In conclusion, we showed that epigenetic silencing by DNA methylation is an unlikely mechanism for PALB2 inactivation in high-grade serous ovarian cancers. Our findings and those of The Cancer Genome Atlas Research Network [2] now challenge the contribution of PALB2 methylation to the dysfunction of the BRCA/FA pathway in this histological subtype of ovarian cancer.
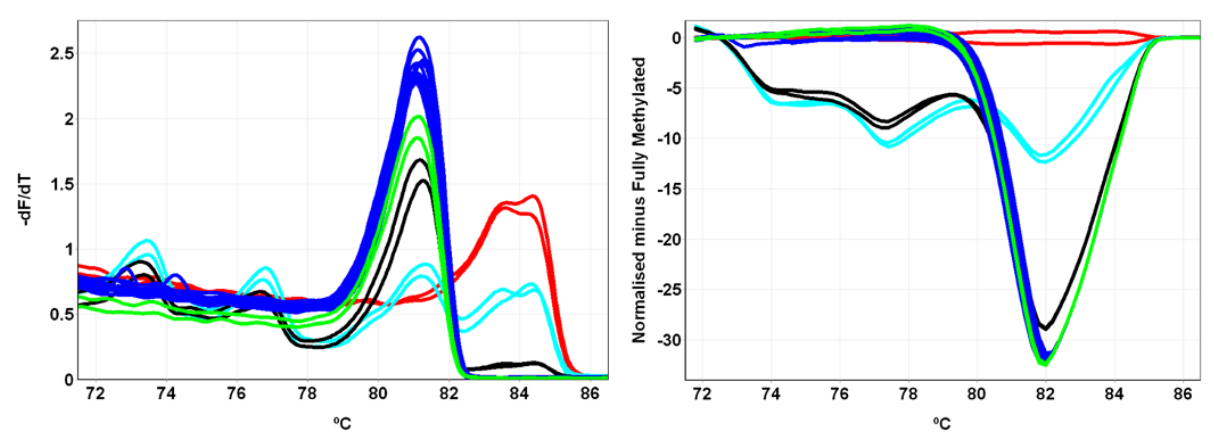

Figure 2 Melting profiles of selected high-grade serous ovarian cancer samples obtained from MS-HRM experiments for PALB2 methylation analysis. The melting profiles were partly smoothened by applying a light digital filter available in the Rotor-Gene 6000 analysis software. Melting profiles for fully methylated (100\%), 50\%, 10\% and unmethylated (0\%) standards as well as the tumour samples are shown as red, turquoise, black, green and blue curves. Tm plots (negative first derivative of the melting curves) are shown in the left panel and the corresponding difference plots are shown in the right panel, respectively. In difference plots, the melting profiles of the fully methylated standard are chosen as a baseline and the relative differences in melting profiles of all other samples are plotted relative to this baseline. The high-grade serous ovarian cancer DNAs (blue curves) are unmethylated for PALB2 as can be seen by their superimposition on the unmethylated standard (green curves). 


\section{Abbreviations}

BRCA/FA: BRCA/Fanconi anaemia; HRM: High-resolution melting; MSHRM: Methylation-sensitive high-resolution melting; PCR: Polymerase chain reaction.

\section{Competing interests}

The authors declare that they have no competing interests.

\section{Authors' contributions}

TM developed the PALB2 MS-HRM assay, performed the experiments, analysed and interpreted the data, and drafted the manuscript. KA performed the experiments, analysed the data, and contributed to the writing of the manuscript. DDLB and GM initiated the Genotyping in the Australian Ovarian Cancer Study project as well as the overarching project of which this investigation forms a part and contributed to the writing of the manuscript. AD supervised the work and co-wrote the manuscript. All authors read and approved the manuscript.

\section{Authors' information}

David DL Bowtell and Alexander Dobrovic are joint senior authors.

\section{Acknowledgements}

$A D$ received funding from the National Breast Cancer Foundation Collaborative Breast Cancer Research Grant Program (CG-08-07) and the Cancer Council of Victoria. The Australian Ovarian Cancer Study was supported by the U.S. Army Medical Research and Materiel Command under DAMD17-01-1-0729, The Cancer Council Tasmania, The Cancer Foundation of Western Australia, the National Health and Medical Research Council of Australia (NHMRC; ID400413), and was approved by the Human Research Ethics Committees at the Peter MacCallum Cancer Centre, Queensland Institute of Medical Research, University of Melbourne and all participating hospitals. The Genotyping in the Australian Ovarian Cancer Study project was supported by the Ovarian Cancer Research Program of the US Department of Defense (W81XWH-08-1-0684 and W81XWH-08-1-0685) (DDLB and GM), Cancer Australia (DDLB and GM) and the National Breast Cancer Foundation (ID509303) (GM), the Peter MacCallum Cancer Centre Foundation (GM) and the Cancer Council Victoria (postgraduate scholarship for KA). We gratefully acknowledge the cooperation of the participating institutions in Australia, and also acknowledge the contribution of the study nurses, research assistants and all clinical and scientific collaborators. The complete AOCS Study Group can be found at www.aocstudy.org. We would like to thank all of the women who participated in the study.

\section{Author details}

'Molecular Pathology Research and Development Laboratory, Department of Pathology, Peter MacCallum Cancer Centre, East Melbourne, VIC, Australia. ${ }^{2}$ Department of Pathology, The University of Melbourne, Parkville, VIC, Australia. ${ }^{3}$ Cancer Genomics and Genetics, Peter MacCallum Cancer Centre, East Melbourne, VIC, Australia. ${ }^{4}$ Department of Biochemistry and Molecular Biology, The University of Melbourne, Parkville, VIC, Australia. ${ }^{5}$ Familial Cancer Centre, Peter MacCallum Cancer Centre, East Melbourne, VIC, Australia. ${ }^{6}$ Sir Peter MacCallum Department of Oncology, The University of Melbourne, Parkville, VIC, Australia.

Received: 10 January 2013 Accepted: 23 March 2013 Published: 12 April 2013

\section{References}

1. Bowtell DD: The genesis and evolution of high-grade serous ovarian cancer. Nat Rev Cancer 2010, 10:803-808.

2. Cancer Genome Atlas Research Network: Integrated genomic analyses of ovarian carcinoma. Nature 2011, 474:609-615.

3. Alsop K, Fereday S, Meldrum C, DeFazio A, Emmanuel C, George J, Dobrovic A, Birrer MJ, Webb PM, Stewart C, Friedlander M, Fox S, Bowtell D, Mitchell G: BRCA mutation frequency and patterns of treatment response in BRCA mutation-positive women with ovarian cancer: a report from the australian ovarian cancer study group. J Clin Oncol 2012, 30:2654-2663.

4. Turner N, Tutt A, Ashworth A: Hallmarks of 'BRCAness' in sporadic cancers. Nat Rev Cancer 2004, 4:814-819.
5. Banerjee S, Kaye S: PARP inhibitors in BRCA gene-mutated ovarian cancer and beyond. Curr Oncol Rep 2011, 13:442-449.

6. Tischkowitz M, Xia B: PALB2/FANCN: recombining cancer and fanconi anemia. Cancer Res 2010, 70:7353-7359.

7. Dansonka-Mieszkowska A, Kluska A, Moes J, Dabrowska M, Nowakowska D, Niwinska A, Derlatka P, Cendrowski K, Kupryjanczyk J: A novel germline PALB2 deletion in Polish breast and ovarian cancer patients. BMC Med Genet 2010, 11:20.

8. Prokofyeva D, Bogdanova N, Bermisheva M, Zinnatullina G, Hillemanns P, Khusnutdinova E, Dörk T: Rare occurrence of PALB2 mutations in ovarian cancer patients from the Volga-Ural region. Clin Genet 2012, 82:100-101.

9. Potapova A, Hoffman AM, Godwin AK, Al-Saleem T, Cairns P: Promoter hypermethylation of the PALB2 susceptibility gene in inherited and sporadic breast and ovarian cancer. Cancer Res 2008, 68:998-1002.

10. Wojdacz TK, Dobrovic A: Methylation-sensitive high resolution melting (MS-HRM): a new approach for sensitive and high-throughput assessment of methylation. Nucleic Acids Res 2007, 35:e41.

11. Mikeska T, Dobrovic A: Methylation-sensitive high resolution melting for the rapid analysis of DNA methylation. In Epigenetics: a reference manual. Edited by Craig JM, Wong NC. Norwich, UK: Caister Academic Press; 2011:325-335.

12. Merritt MA, Green AC, Nagle CM, Webb PM, Australian Cancer Study (Ovarian Cancer) Australian Ovarian Cancer Study Group: Talcum powder, chronic pelvic inflammation and NSAIDs in relation to risk of epithelial ovarian cancer. Int J Cancer 2008, 122:170-176.

13. Mikeska T, Felsberg J, Hewitt CA, Dobrovic A: Analysing DNA methylation using bisulphite pyrosequencing. Methods Mol Biol 2011, 791:33-53.

14. Huang KT, Dobrovic A, Yan M, Karim RZ, Lee CS, Lakhani SR, Fox SB: DNA methylation profiling of phyllodes and fibroadenoma tumours of the breast. Breast Cancer Res Treat 2010, 124:555-565.

15. Montavon C, Gloss BS, Warton K, Barton CA, Statham AL, Scurry JP, Tabor B, Nguyen TV, Qu W, Samimi G, Hacker NF, Sutherland RL, Clark SJ, O'Brien PM: Prognostic and diagnostic significance of DNA methylation patterns in high grade serous ovarian cancer. Gynecol Oncol 2012, 124:582-588.

16. Mikeska T, Candiloro IL, Dobrovic A: The implications of heterogeneous DNA methylation for the accurate quantification of methylation. Epigenomics 2010, 2:561-573.

17. Candiloro IL, Mikeska T, Dobrovic A: Closed-tube PCR methods for locus-specific DNA methylation analysis. Methods Mol Biol 2011, 791:55-71.

18. Zorn KK, Bonome T, Gangi L, Chandramouli GV, Awtrey CS, Gardner GJ, Barrett JC, Boyd J, Birrer MJ: Gene expression profiles of serous, endometrioid, and clear cell subtypes of ovarian and endometrial cancer. Clin Cancer Res 2005, 11:6422-6430.

19. Bell DA: Origins and molecular pathology of ovarian cancer. Mod Pathol 2005, 18(Suppl 2):S19-S32.

20. Kurman RJ, Shih IM: The origin and pathogenesis of epithelial ovarian cancer: a proposed unifying theory. Am J Surg Pathol 2010, 34:433-443.

21. Anglesio MS, George J, Kulbe H, Friedlander M, Rischin D, Lemech C, Power J, Coward J, Cowin PA, House CM, Chakravarty P, Gorringe KL, Campbell IG, Australian Ovarian Cancer Study Group, Okamoto A, Birrer MJ, Huntsman DG, de Fazio A, Kalloger SE, Balkwill F, Gilks CB, Bowtell DD: IL6-STAT3-HIF signaling and therapeutic response to the angiogenesis inhibitor sunitinib in ovarian clear cell cancer. Clin Cancer Res 2011, 17:2538-2548.

doi:10.1186/1757-2215-6-26

Cite this article as: Mikeska et al:: No evidence for PALB2 methylation in high-grade serous ovarian cancer. Journal of Ovarian Research 2013 6:26. 